\title{
Data-driven analysis of simultaneous EEG/fMRI using an ICA approach
}

\section{Lena Schmüser $^{1 *}$, Alexandra Sebastian ${ }^{1}$, Arian Mobascher ${ }^{1}$, Klaus Lieb ${ }^{1}$, Oliver Tüscher ${ }^{1,2,3}$ and Bernd Feige ${ }^{2}$}

' Emotion Regulation and Impulse Control Group, Focus Program Translational Neuroscience, Department of Psychiatry and Psychotherapy, Johannes Gutenberg University of Mainz, Mainz, Germany

2 Department of Psychiatry and Psychotherapy, Albert Ludwigs University of Freiburg, Freiburg, Germany

${ }^{3}$ Department of Neurology, Albert Ludwigs University Medical Center, Freiburg, Germany

\section{Edited by:}

Pedro Antonio Valdes-Sosa, Cuban

Neuroscience Center, Cuba

\section{Reviewed by:}

Urs Maurer, University of Zurich, Switzerland

Tomas Ossandon, Pontificia

Universidad Catolica de Chile, Chile

${ }^{*}$ Correspondence:

Lena Schmüser, Emotion Regulation and Impulse Control Group, Focus

Program Translational Neuroscience, Department of Psychiatry and

Psychotherapy, Johannes Gutenberg

University of Mainz, Untere

Zahlbacher Strasse 8, 55131 Mainz,

Germany

e-mail: lena.schmueser@

unimedizin-mainz.de
Due to its millisecond-scale temporal resolution, EEG allows to assess neural correlates with precisely defined temporal relationship relative to a given event. This knowledge is generally lacking in data from functional magnetic resonance imaging (fMRI) which has a temporal resolution on the scale of seconds so that possibilities to combine the two modalities are sought. Previous applications combining event-related potentials (ERPs) with simultaneous $\mathrm{FMRI}$ BOLD generally aimed at measuring known ERP components in single trials and correlate the resulting time series with the fMRI BOLD signal. While it is a valuable first step, this procedure cannot guarantee that variability of the chosen ERP component is specific for the targeted neurophysiological process on the group and single subject level. Here we introduce a newly developed data-driven analysis procedure that automatically selects task-specific electrophysiological independent components (ICs). We used single-trial simultaneous EEG/fMRI analysis of a visual Go/Nogo task to assess inhibition-related EEG components, their trial-to-trial amplitude variability, and the relationship between this variability and the fMRI. Single-trial EEG/fMRI analysis within a subgroup of 22 participants revealed positive correlations of $\mathrm{FMRI} B O L D$ signal with EEG-derived regressors in fronto-striatal regions which were more pronounced in an early compared to a late phase of task execution. In sum, selecting Nogo-related ICs in an automated, single subject procedure reveals fMRI-BOLD responses correlated to different phases of task execution. Furthermore, to illustrate utility and generalizability of the method beyond detecting the presence or absence of reliable inhibitory components in the EEG, we show that the IC selection can be extended to other events in the same dataset, e.g., the visual responses.

Keywords: single-trial EEG/fMRI, trial-to-trial variability, independent component analysis, response inhibition, Go/Nogo, visual response

\section{INTRODUCTION}

Behavioral variability such as the varying effectiveness of motor inhibition or trial-to-trial variations of reaction times has been linked to single-trial variabilities in neural processes (MacDonald et al., 2006; Fontanini and Katz, 2008; Ledberg et al., 2012). It has been shown that single-trial variability of evoked neural activity can be modeled by a combination of random ongoing network activity and stationary stimulus-related responses (Arieli et al., 1996). Therefore, single-trial fluctuations of evoked responses contain aspects of moment-to-moment fluctuations in the participant's brain state rather than only representing noise (Lutz et al., 2002; Kelly et al., 2008; Ledberg et al., 2012). Assessing trial-to-trial variations is also key to characterize intra- as well as inter-individual behavioral and neural processing phenotypes.

In order to meaningfully analyze trial-to-trial variations, they have to be related to other parameters available for the same single trials. Trial-by-trial coupling of simultaneous EEG/fMRI data (Debener et al., 2005; Eichele et al., 2005; Huster et al.,
2012) allows relating single-trial event-related EEG with singletrial BOLD data in an attempt to map the changes in the participant's brain state mentioned above to changes in metabolic brain activity. This approach is also designated "integration-byprediction" regarding that single-trial EEG/fMRI analyses usually employ EEG-derived regressors as predictors of the fMRI BOLD responses (for more detail see Debener et al., 2006; Eichele et al., 2009; Bland et al., 2011). In the process of isolating taskrelated single-trial EEG activity, different routines have been used: single-trial EEG features are extracted from single independent components (ICs) reflecting best the EEG component of interest (Debener et al., 2005; Feige et al., 2005; Mobascher et al., 2009), from artifact-cleaned EEG data using several electrodes (Eichele et al., 2005; Novitskiy et al., 2011) or single electrodes (Bénar et al., 2007; Mulert et al., 2008; Warbrick et al., 2009; Karch et al., 2010; Scheibe et al., 2010; Juckel et al., 2012; Baumeister et al., 2014). However, in the majority of studies components of interest were identified by visual inspection, which depends on subjective 
evaluation and can be biased by inter- and intra-individual variations of the evaluator.

Greater objectivity can instead be provided by data-driven approaches of component selection. Goldman et al. (2009) for example selected task-discriminating EEG components in a completely data driven way. The authors identified taskrelated components which discriminated two task conditions in stimulus-locked and response-locked time windows. They demonstrated that single-trial correlations of these taskdiscriminating components with fMRI BOLD responses could reveal brain areas different from those yielded by classical fMRI analyses. However, an important limitation of this linear discrimination method is that the algorithm could extract only one EEG component for a given time window and thus could miss meaningful components. Another approach that circumvents this by selecting the components in source space instead of sensor space was presented by Wessel and Ullsperger (2011). The authors developed an algorithm (COMPASS) that automatically identifies ICs contributing to the event-related potential (ERP) of interest by comparing each IC with a predefined ERP template. This approach however is limited by restricting the selection procedure to predefined ERP templates. Thus, we aimed to develop a new approach enabling us to classify and select inhibitionrelated ICs intra-individually in a data driven way without a priori implicating known ERP components. This approach avoids the analytical bias of the assessment of EEG correlates of task-related activity introduced by the restriction to distinct ERP components. Furthermore, by classifying and selecting ICs intra-individually our approach takes into account known inter-individual differences in neural processing (Kanai and Rees, 2011).

This automated procedure was applied to simultaneously acquired EEG and fMRI data of 39 healthy control participants who had performed a visual Go/Nogo task. This paradigm has been used in several EEG/fMRI studies, including studies investigating the effect of fMRI data acquisition on ERP. Bregadze and Lavric (2006), for instance, showed that Nogo-related ERP components can be extracted from EEG data recorded simultaneously with fMRI data acquisition. In 2011, Lavric et al. demonstrated that the detection of task-related modulations of N2/P3 ERPs could be improved by applying ICA-based analyses. Variants of the classical Go/Nogo task were also used in simultaneous EEG/fMRI studies in order to assess the neural correlates of response inhibition. Using a cued auditory Go/Nogo task during simultaneous EEG/fMRI, Karch et al. (2008) found correlations of fMRI BOLD signal in insular, right temporo-parietal and medial frontal cortex with fronto-central Nogo-P3 amplitude values. Most recently, Baumeister et al. (2014) investigated the role of N2 and $\mathrm{P} 3$ in cognitive processes associated with response inhibition by using parametric modulation of fMRI BOLD signal with both $\mathrm{N} 2$ and P3 single-trial amplitude values derived from Cz. This analysis revealed an association of $\mathrm{N} 2$ with attentional processes while P3 was associated with inhibitory processes but also with memory recollection and internal reflection (Baumeister et al., 2014). Although the above studies hint at a relation of the N2/P3 complex to Go/Nogo inhibitory processes it is not clear how specific variations of the N2/P3 complex are for inhibition. Thus, instead of selecting a-priori defined distinct ERP components such as the N2/P3 complex we used a purely data-driven approach to select ICs reliably associated with inhibition.

The present study was designed to examine the BOLD correlates of variations in electrophysiological inhibition-related components in a data-driven approach. While previous studies used fixed latency windows and distinct EEG channels to derive regressors from the EEG, relying upon data from other EEG studies or own grand averages for their choice, we automatically selected IC components reliably associated with response inhibition for each single participant. We ensured to use only ICs which had reliably larger amplitude in Nogo than in Go trials securing the specificity of EEG components for neural activity of response inhibition. We therefore introduce a newly developed data-driven analysis procedure that automatically selects participant-specific electrophysiological ICs which are reliably and specifically Nogo-related at an early or late stage of response inhibition to inform fMRI data analysis. To assess and validate the performance and outcome of our automated procedure in the context of combined EEG/fMRI analysis procedures, we compared our automated ICbased approach to an approach based on selecting single-trial amplitude values from predefined ERP components (see Figure 1 for a graphical overview). Thus, in line with Baumeister et al. (2014) we extracted for each participant the mean amplitude values of N2 (280-340 ms post-stimulus) and P3 (350-570 ms post-stimulus) from the $\mathrm{Cz}$ site. Second, to illustrate the utility of the method beyond detecting Nogo-related components, we used the same data-driven analysis procedure for detecting visual responses in the same dataset. Visual components are well suited for validation purpose, as consistent results have been found in previous EEG/fMRI studies with different task settings (Di Russo et al., 2002, 2005; Novitskiy et al., 2011; Warbrick et al., 2013). Using separate EEG and fMRI data acquisition, Di Russo et al. (2002, 2005) showed that the P1 and N1 subcomponents can be accounted for by dipoles localized to middle occipital gyrus, fusiform gyrus and parietal lobe. More recently Novitskiy et al. (2011) and Warbrick et al. (2013) found positive singletrial correlations of visual components with fMRI BOLD signal in regions of the visual dorsal stream but also in medial frontal and precentral gyri.

\section{MATERIALS AND METHODS}

Taking advantage of the fact that independent component analysis (ICA) can be used to isolate task-related components (Debener et al., 2005; Bagshaw and Warbrick, 2007), we employed ICA in order to selectively extract time series related to different phases of task execution of a visual Go/Nogo task. Importantly, the algorithm we used was not designed to identify ICs associated with classical event-related components such as N2 and P3, but to automatically select ICs with significantly increased amplitudes in Nogo trials compared to Go trials within predefined time windows located prior and around the individual's median response time (RT). Thus, for each participant, EEG data were decomposed into temporally ICs which were then intra-individually classified according to their relation to response inhibition (Nogo > Go). ICs identified as reliably Nogo-related within a predefined time window were combined into individual electrophysiological regressors and then included into fMRI first-level analysis. 


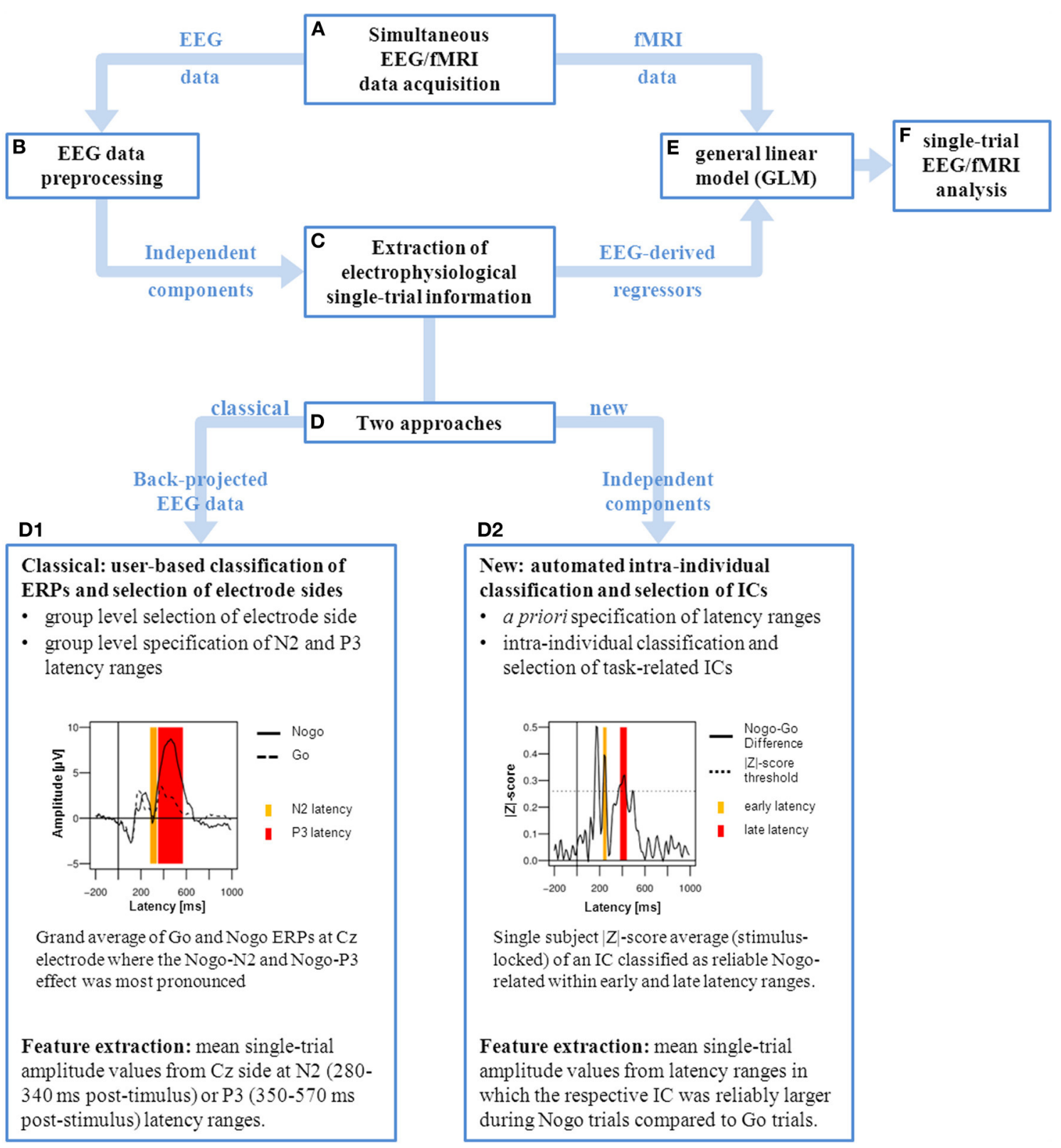

FIGURE 1 | Graphical representation of single-trial EEG/fMRI analysis. After simultaneous EEG/fMRI data acquisition (A) the EEG data is preprocessed and corrected for fMRI artifacts (B) using independent component analysis (ICA). Subsequently the electrophysiological single-trial values can be extracted (C) using different approaches (D). Classically (D1), single-trial amplitude values are extracted from predefined ERP components. This is based on a chosen electrode site where the ERP component of interest (Nogo-N2 and Nogo-P3) is most pronounced in the grand mean average. Followed by the specification of N2 (280-340 ms, yellow) and P3 (350-570 ms, red) latency ranges which cover best the task-related ERP effects on group level at the selected electrode site (Cz). For each participant the mean single-trial values are extracted from these predefined latency

\section{EXPERIMENTAL DESIGN \\ Participants}

Thirty-nine participants (16 males; mean age: $38.85 \pm 16.48$ ) were included in this analysis. Participants were recruited from ranges. Alternatively (D2), our approach allows to extract single-trial values from independent components (ICs) which are intra-individually classified and selected in an automated procedure. This is based on a priori specification of latency ranges of interest, in this case located prior (early, yellow) and around (late, red) the individual's median response time (RT). ICs are intra-individually classified according their association with the Nogo condition (significantly increased amplitudes in Nogo trials compared to Go trials). For each participant the mean single-trial values are extracted from latency ranges in which the respective IC was reliably larger during Nogo. In both approaches the resulting electrophysiological regressors are included in the general linear model of $f M R I$ data analysis (E) in order to perform the single-trial EEG/fMRI data analysis (F)

a larger sample (Sebastian et al., 2013a) because of their good overall data quality for EEG and fMRI (see section Data Preprocessing). All participants were right-handed (Oldfield, 1971) and had normal or corrected-to-normal vision. Structural 
Clinical Interview for DSM-IV Axis I and II Disorders (SCIDI/II) was used (Wittchen et al., 1997) to exclude participants with a lifetime history of axis I or axis II disorders. The study was approved by the Ethics Committee of the University of Freiburg Medical School and all participants gave their informed consent prior to MRI scanning. Each participant received a financial compensation of $€ 55$.

\section{Experimental paradigm}

During the simultaneous EEG/fMRI session all participants performed a visual Go/Nogo-task. Participants were instructed to respond by pressing a mouse button with the right index finger to every letter (Go stimulus) except for the letter " $\mathrm{X}$ " (Nogo stimulus). Each letter was shown for $500 \mathrm{~ms}$ followed by a black screen for the next $500 \mathrm{~ms}$. Every participant completed two runs each consisting of 300 stimuli. Nogo stimuli were presented with a mean probability of $29 \%$, and each Nogo stimulus was followed by at least one Go stimulus (Sebastian et al., 2013a,b, 2012). Visual stimuli were programmed using the software "Presentation" (Neurobehavioral Systems, Version 11.1 http://www.neurobs.com/) and were projected on a screen at the head end of the scanner bore and viewed with the aid of a mirror mounted on the head coils. Participants had in advance received a brief training session on the task outside the scanner room.

\section{DATA ACQUISITION}

The study was conducted at the University Hospital of Freiburg (Department of Radiology). fMRI data acquisition and EEG recordings were initiated manually whereas visual presentation was initiated by a trigger code sent from the MR scanner. The EEG-amplifier hardware clock was synchronized with the timing of gradient switching during fMRI measurements (SyncBox; Brain Products, Gilching, Germany). Onsets of stimulation and echo-planar image (EPI) scans as well as the participant's response were registered on a trigger channel of the EEG acquisition host.

\section{fMRI/MRI}

MRI data was collected using a 3T tim-TRIO scanner (Siemens Medical Systems, Erlangen) equipped with a 12 channel head coil. Foam padding was used to limit head motion within the coil. For functional BOLD imaging, T2*-weighted EPI volumes were acquired $\left(T R=2250 \mathrm{~ms}, T E=30 \mathrm{~ms}\right.$, flip angle $=90^{\circ}$, $F O V=92 \mathrm{~mm}$, voxel size $=3 \times 3 \times 3 \mathrm{~mm}, 36$ slices) by applying fully automated PACE (Prospective Acquisition Correction) motion correction (Thesen et al., 2000) and distortion correction based on point spread function mapping (Zaitsev et al., 2004). Per run 157 complete brain volumes were acquired. Following fMRI data acquisition, the EEG cap was removed and 3D MRI data for anatomical references was acquired using a 3D magnetization prepared, rapid acquisition gradient echo (MPRAGE) sequence $(T R=2200 \mathrm{~ms}, T E=4.11 \mathrm{~ms}$, flip angle $=$ $12^{\circ}, F O V=256 \mathrm{~mm}$, voxel size $\left.=1 \times 1 \times 1 \mathrm{~mm}\right)$.

\section{EEG}

Continuous EEG data was recorded with a 64-channel EEG-system consisting of two 32-channel MR compatible EEG-amplifiers (BrainAmp MR plus; Brain Products) powered by a MR-compatible rechargeable battery pack (PowerPack, Brain Products). The EEG system was placed inside the scanner bore directly behind the head coil. This allowed for the use of short wires, thus reducing potential scanner artifacts caused by wires moving inside the magnetic fields. A total of 62 sintered $\mathrm{Ag} / \mathrm{AgCl}$ ring electrodes were placed within an elastic EEG-recording cap (EasyCap, Falk Minow Services, Herrsching, Germany). Electrodes were placed according to an extended international 10-20 system with reference electrode positioned at $\mathrm{FCz}$ and ground electrode positioned at AFFz. In order to monitor electrocardiograms (ECG) and eye blinks (EOG), additional electrodes were placed beneath the participant's left scapula and below the left eye. Electrode-skin contact impedances were maintained below $10 \mathrm{k} \Omega$. The recorded analog EEG signal was filtered between DC and $1 \mathrm{kHz}$, digitized with a sampling frequency of $5 \mathrm{kHz}$ and transmitted via fiber optic cables to a recording PC placed outside the scanner room. To facilitate subtraction of the gradient artifact, EEG sampling was driven by the clock board of the MR scanner (SyncBox, Brain Products). The Brain Vision Recorder software (Brain Products) was used to acquire, store and display EEG recordings online.

\section{DATA PREPROCESSING}

\section{fMRI preprocessing}

Image preprocessing was performed using SPM5 (Wellcome Trust Center for Neuroimaging at UCL, London, UK; http://www.fil. ion.ucl.ac.uk/spm/software/spm5) running under Matlab 7.7.0 (The MathWorks Inc., Natick, Massachusetts, USA; http://www. mathworks.com). Images were screened for motion artifacts prior to data analysis. No excessive head motion ( $>2 \mathrm{~mm}$ ) was observed in any of the subjects. Next, images were manually reoriented to the T1-template of SPM. To allow for equilibrium effects the first five volumes of each run were discarded. Functional images were then realigned to the first image of the first run (six degreesof-freedom rigid body transformation) and coregistered to the individual T1. The T1 image was then spatially normalized (linear and non-linear transformation) into the reference system of the Montreal Neurological Institute's (MNI) reference brain. Functional images were spatially normalized using the resultant normalizing parameters and then smoothed by applying a $3 \mathrm{D}$ isotropic Gaussian kernel ( $8 \mathrm{~mm}$ full-width at half maximum, FWHM).

\section{EEG preprocessing}

EEG data was processed offline using AvgQ (Feige, 1999; Freiburg, Germany; https://github.com/berndf/avg_q), an open source multichannel (EEG/MEG) data processor driven by Python scripts. Gradient artifact correction was performed by template subtraction (Allen et al., 2000). Data upsampling was not necessary since the EEG sampling was synchronized to the gradient clock (SyncBox). In order to remove low-frequency drifts as well as residual scanner artifacts, the gradient-corrected EEG data was then run through a bandpass filter $(0.2-48 \mathrm{~Hz})$ and down-sampled to $100 \mathrm{~Hz}$. Afterwards, an unmixing matrix was estimated using the extended infomax algorithm (ICA, Bell and Sejnowski, 1995; Lee et al., 1999; Makeig et al., 1999, 2002) for the continuous EEG data of each participant and run separately. 
By multiplying the continuous EEG data with this unmixing matrix, IC activations can be computed for any EEG sample without repeating the ICA training. For ballistocardiographic (BCG) and electrooculographic (EOG) artifact detection and correction, averages related to heart beat and eye blink were computed. Single heart beats were detected in the ECG signal by convolution with a time-domain ECG template. The continuous EEG was averaged with respect to the detected heart beats and ICs loading on this average identified. Artifact correction was performed by removing these ICs. Based on the BCG artifact corrected EEG data, EOG artifact detection and correction was performed similarly. Importantly, BCG/EOG artifact corrected EEG is not used in the main method illustrated here. In this method, the electrophysiological information used for single-trial EEG/fMRI analysis is extracted from the IC time courses themselves, excluding those ICs representing BCG/EOG artifacts. Participants of an initially larger sample (Sebastian et al., 2013a) of whom the artifact correction did not achieve usable datasets were excluded from further analysis.

\section{CLASSIFICATION AND SELECTION OF INDEPENDENT COMPONENTS}

The IC time courses of each participant were segmented into epochs of $1200 \mathrm{~ms}$ starting $200 \mathrm{~ms}$ prior to stimulus onset. The $200 \mathrm{~ms}$ pre-stimulus interval was used for baseline correction. Epochs belonging to the same event type (i.e., correct response: Go and Nogo; incorrect response: omission of Go trials and commission errors in Nogo trials) were averaged, resulting in four different event-related averages each consisting of 64 averaged ICs.

\section{Reliability testing and thresholding}

In addition to the pointwise mean, pointwise variance information was collected. Together they were used to compute pointwise $t$-tests comparing either the Nogo and Go conditions (twosample $t$-test for independent groups) or comparing the average against baseline (one-sample $t$-test) on each IC and event type. In a second step, the point-wise $t$-values were transformed into $Z$-scores as basis for the subsequent IC classification. As high $Z$ scores are indicative of high signal-to-noise ratios, only ICs with absolute $Z$-scores crossing a predefined threshold entered further analysis steps. Since there were 300 trials per block of which 90 were Nogo trials, we selected a $Z$-score of 0.275 which corresponds to a two-sided $p$-value of 0.01 (two-sample $t$-test with $d f=89$ ), choosing the degree of freedom conservatively from the condition with the smaller number of epochs contributing to the analysis.

\section{Nogo-related ICs}

IC classification. To determine components reliably associated with the Nogo condition, $Z$-score differences between Nogo- and Go-related IC averages were computed as described in section Reliability Testing and Thresholding. Nogo-Go differences were classified as sufficiently reliable if their absolute $Z$-score exceeded a threshold of 0.275 . All latency ranges with above-threshold $Z$ scores were noted, i.e., the latencies at which the threshold was crossed in positive and in negative direction. The polarity of ICs in a given latency range is arbitrary. To be able to select only such

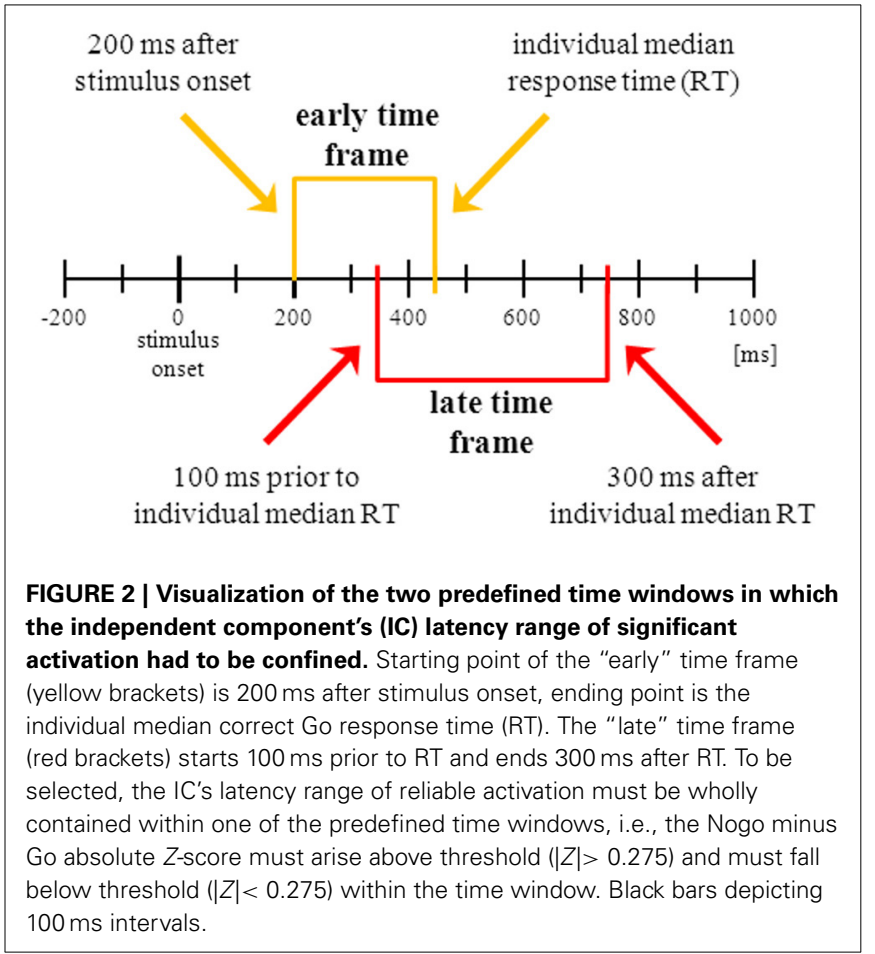

ICs and latency ranges in which the Nogo amplitude was larger, a direction was defined for each latency range by noting the polarity of the event-related IC average with the larger absolute amplitude in this latency range. For each participant, a list of Nogo-related ICs, latency ranges and polarities was formed keeping only those associated with larger absolute amplitudes in Nogo trials.

IC selection. To construct regressors representing different phases of the inhibition processes, we combined ICs and latency ranges falling into different time windows (Figure 2). These time windows were defined for each participant in relation to stimulus onset and the individual's median correct Go RT. To capture neural correlates of an early stage of response inhibition without including correlates of visual processing and object recognition (Johnson and Olshausen, 2003), the "early" time window starts $200 \mathrm{~ms}$ after stimulus onset and ends with the individual median RT (Figure 2, yellow part). To capture neural correlates of a later stage of response inhibition we defined a second time window located around the participant's RT. Considering the trial-to-trial fluctuations of single trial RTs around median RT the "late" time window starts $100 \mathrm{~ms}$ before RT and ends $300 \mathrm{~ms}$ after RT (Figure 2, red part). We allowed overlapping latency windows because both start and end of each acceptable latency range as determined previously (Nogo-Go $|Z|>0.275$ ) were strictly required to fall within the given ranges (Figure 3A). This means that any acceptable activity not only has to be significant within the given time range but must also start (i.e., rise above $Z$-threshold) after the beginning and end (i.e., fall under $Z$-threshold) before the end of the given range. The overlapping time ranges were chosen to avoid losing too many meaningful activity candidates. 
A a Grand average of early and late independent components (ICs)

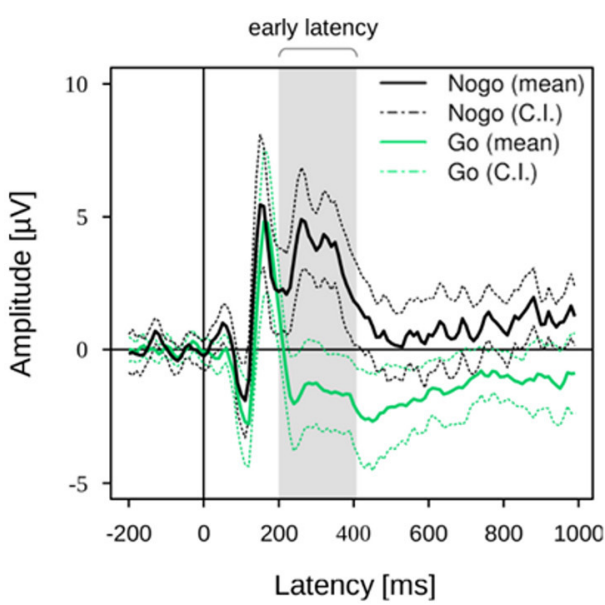

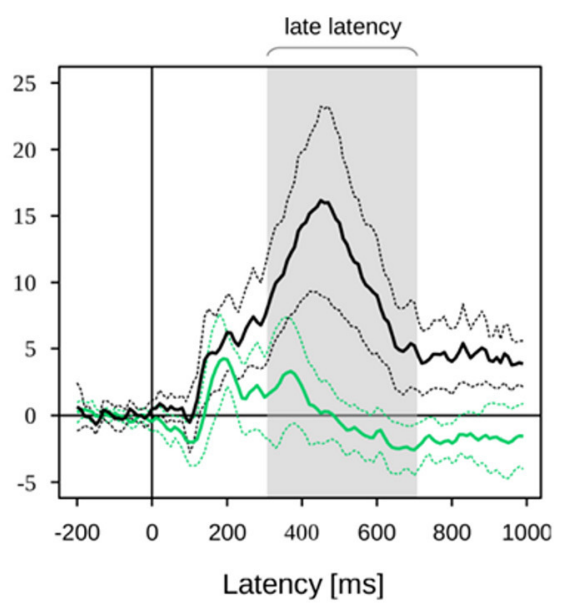

b Topography of
Nogo at early latency

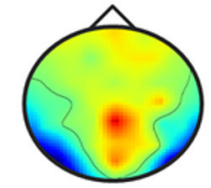

c Topography of Nogo at late latency

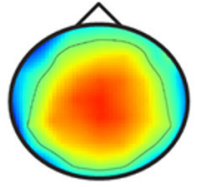

B a Positive single-trial correlations of early ICs with fMRI BOLD signal
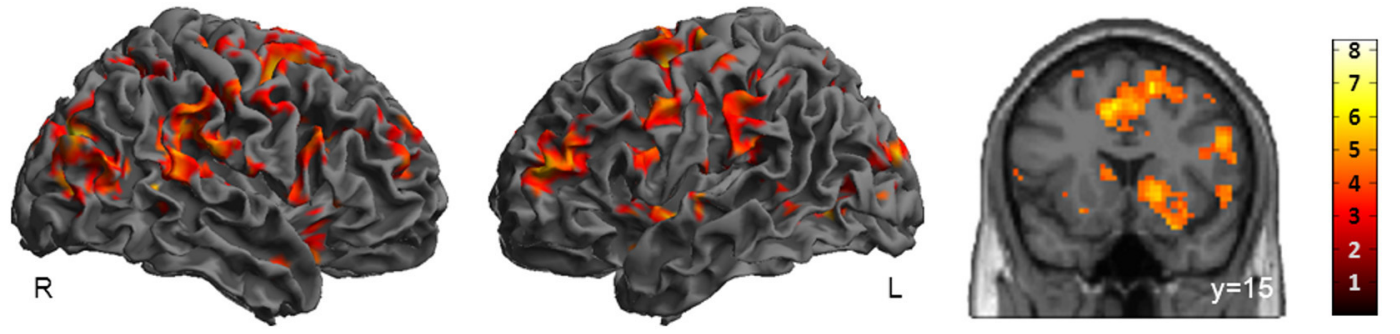

b Positive single-trial correlations of late ICs with fMRI BOLD signal
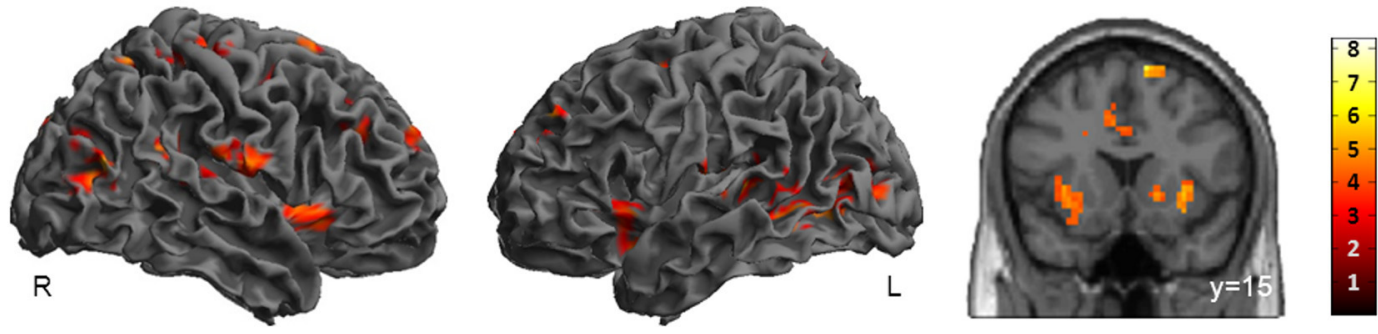

FIGURE 3 | (A) IC activation grand averages (a) and grand mean topographies (b,c) of all participants ICs which were classified as reliably Nogo-related within the early latency range or late latency range. Mean and 95\% confidence interval (Cl) of Nogo (black lines) and Go (green lines) are displayed in solid and dotted lines, respectively; gray bars indicate the early and late latency ranges on the group level. (B) Activation maps displaying the main effects of positive correlations with EEG-derived early (a; time window starting $200 \mathrm{~ms}$ after stimulus onset and ending with the RT) and late (b; time window starting $100 \mathrm{~ms}$ prior to RT and ending $300 \mathrm{~ms}$ after RT) regressors. These regressors were orthogonalized to onset regressors. Images are displayed in neurological order ( $L$, Left; R, Right), with $p<0.005$ (unc.) and $k=20$. $R T$, individual median Go response time.
Feature extraction. For every selected IC, mean amplitudes of all single trials were extracted from the latency range in which the respective IC component was reliably larger during Nogo and inverted if necessary to ensure positive polarity with respect to the Nogo-Go difference, using the polarity noted above. To be able to compare the time ranges across a single group of participants, participants failing to display Nogo-specific ICs on the chosen absolute $Z$-score level $(|Z|>0.275)$ for any time window were excluded. For each of the remaining 22 participants ( 7 males; mean age: $34.41 \pm 14.1$ ), amplitude vectors of ICs selected with respect to the same time window (early or late) and run were combined into a single amplitude vector by summation. This resulted in two different EEG-derived regressors for each participant and run: early and late.

\section{Visual ICs}

To assess whether component selection can be done on other electrophysiological components in the same dataset of the visual Go/Nogo task, we modified the approach so that visual components can be detected. 
IC classification. In contrast to the Nogo-related ICs which we expected to have larger amplitudes in Nogo than in Go conditions, we assumed that visual responses are similar in Go and Nogo conditions. Thus, instead of for the Nogo-Go difference, IC average and variance were computed across Nogo and Go epochs. ICs were classified as sufficiently reliably activated if their absolute $Z$-score exceeded a threshold of 0.275 . For each participant and run, a list of ICs, latency ranges and polarities was formed.

IC selection. To construct a regressor representing visual processing we combined ICs and latency ranges falling into a time window starting $90 \mathrm{~ms}$ after stimulus onset and ending $140 \mathrm{~ms}$ after stimulus onset (Figure 5A).

Feature extraction. For every selected IC, mean amplitudes of each single trial were extracted from the latency range in which the respective IC component had crossed the predefined $Z$ threshold. Two participants failed to display visual ICs on the chosen absolute $Z$-score level $(|Z|>0.275)$ and were therefore excluded from any further analyses. For each of the remaining 37 participants (15 males; mean age: $38.27 \pm 16.1$ ) and run, amplitude vectors of the selected ICs were combined into a single amplitude vector by summation.

\section{CLASSIFICATION AND SELECTION OF N2/P3 ERPs}

To compare the automated IC-based approach to a more classical approach based on selecting single-trial amplitude values from predefined ERP components, we extracted the mean N2/P3 amplitude values. To achieve improved comparability, single-trial EEG/fMRI analysis of N2/P3 amplitudes was computed for the same 22 participants as the single-trial EEG/fMRI analysis of Nogo-related ICs.

The BCG/EOG artifact corrected EEG of each participant was re-referenced to the average of TP 9 and TP10 and segmented into epochs of $1200 \mathrm{~ms}$ starting $200 \mathrm{~ms}$ prior to stimulus onset. The $200 \mathrm{~ms}$ pre-stimulus interval was used for baseline correction. Epochs belonging to the same event type (i.e., correct response: Go and Nogo; incorrect response: omission of Go trials and commission errors in Nogo trials) were averaged, resulting in four different event-related averages.

For each participant, single-trial amplitude values were extracted from $\mathrm{Cz}$ where the Nogo-N2/-P3 effects were most pronounced in the grand average. N2 was measured as the mean amplitude in the time window 280-340 ms after stimulus onset, whereas P3 was measured as the mean amplitude between 350 and $570 \mathrm{~ms}$ after stimulus. These latency ranges were chosen to cover best the task-related N2 and P3 effects on group level (Figure 4A) Mean amplitudes of each single trial were extracted from the $\mathrm{N} 2$ and $\mathrm{P} 3$ latency ranges at $\mathrm{Cz}$, resulting in two amplitude vectors for each participant.

\section{fMRI REGRESSORS}

To fit the sampling frequencies of EEG-derived regressors $(f=$ $1 \mathrm{~Hz})$ and $\mathrm{fMRI}$ data acquisition $(f=1 / 2.25 \mathrm{~Hz})$, each joint amplitude vector was interpolated over time by using a cubic smoothing spline function and re-sampled at the time points of fMRI data acquisition. This down-sampled time course was then normalized to inter-quartile range $(\mathrm{IQR}=1)$ and convolved with a canonical hemodynamic response function. In a second step, each EEG-derived regressor was orthogonalized with respect to classical onset regressors (Go, Nogo, Errors).

\section{FIRST-LEVEL SINGLE-TRIAL EEG/fMRI ANALYSIS}

Statistical analysis of fMRI data was performed using SPM8 running under Matlab 7.7.0. For each participant $(N=37$ in case of visual components and $N=22$ in all other cases) different GLMs were fitted separately to the fMRI data. The design matrix of each GLM contained four regressors of interest: three onset regressors (Go, Nogo, and Errors) and one EEG regressor derived from either early or late ICs, N2 or P3 ERP, or visual ICs. Task-related EEG-derived regressors were orthogonalized to the onset regressors, whereas the visual regressor was not orthogonalized. The time courses of regressors and functional data were run through a high-pass filter with a $128 \mathrm{~s}$ cut-off in order to remove artifacts resulting from low frequency temporal variations. Since ICA was applied to each run independently, we obtained two EEG-derived regressors and thus two corresponding contrast images for each participant and time window. A single beta image per participant was computed for second-level analyses by averaging the two contrast images belonging to the same participant and time window.

\section{GROUP ANALYSIS}

The first level analysis results of task-related regressors (i.e., early/late and N2/P3) were subjected to paired $t$-tests with either early/late or N2/P3 as paired observations. The first level analysis results of EEG regressors derived from visual components were subjected to a one-sample $t$-test. For whole brain analysis the statistic images were assessed for cluster-wise significance by using a cluster-defining height threshold of $p<0.05$ (familywise error $=$ FWE correction for multiple comparisons). In the case of region of interest (ROI) analyses, clusters were assessed for peak-wise significance by using a height threshold of $p<$ 0.05 (FWE corrected). Following Sebastian et al. (2013b), small volume correction was computed for the following predefined ROIs as derived from the automated anatomical labeling atlas $(\mathrm{AAL})$ : lateral inferior frontal cortex (IFC; derived from a combination of pars opercularis and pars triangularis); middle frontal gyrus; pre-supplementary motor area (preSMA; derived from the SMA region with $y>0$ ); caudate nucleus; putamen and pallidum. Additionally small volume correction was computed for the subthalamic nucleus (STN), consisting of two boxes of respectively $10 \times 10 \times 10 \mathrm{~mm}$ in size and localized at the MNI coordinates $-10,-15,-5$ (left STN) and 10, $-15,-5$ (right STN) (Aron and Poldrack, 2006). For visual components, bilateral inferior occipital cortex, bilateral middle occipital cortex and bilateral superior occipital cortex were additionally included.

\section{RESULTS}

\section{EEG/fMRI SINGLE-TRIAL ANALYSIS OF NOGO-RELATED ICs}

Positive correlations of the fMRI BOLD signal with EEG regressors derived from IC related to task processing at an early (200 ms after stimulus onset until individual median RT) and later $(100 \mathrm{~ms}$ prior to median RT until $300 \mathrm{~ms}$ after median RT) stage of response inhibition are listed in Table 1. Due to 


\section{A a Grand average at $\mathrm{Cz}$ electrode}

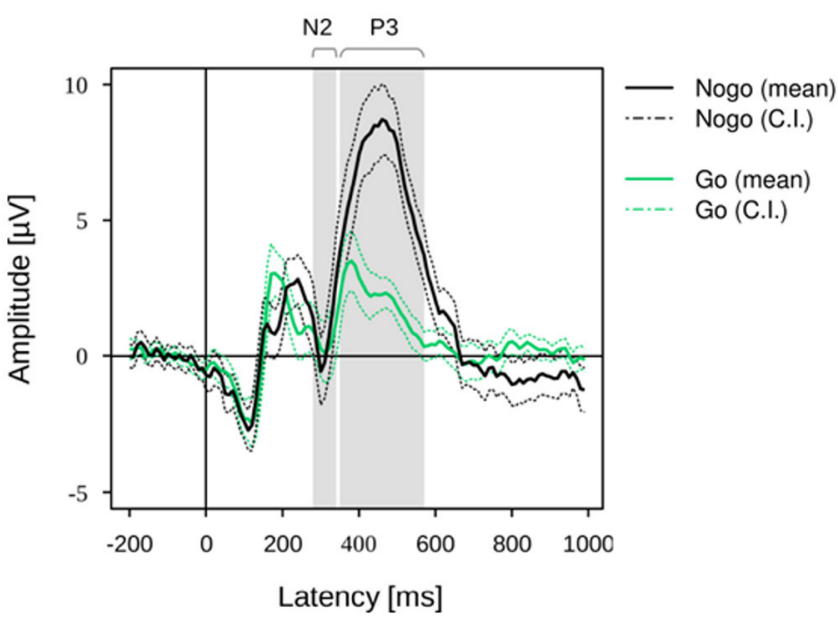

b Topography of Nogo at N2

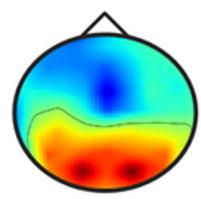

c Topography of Nogo at P3

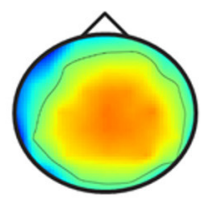

B a Positive single-trial correlations of N2 at Cz with fMRI BOLD signal
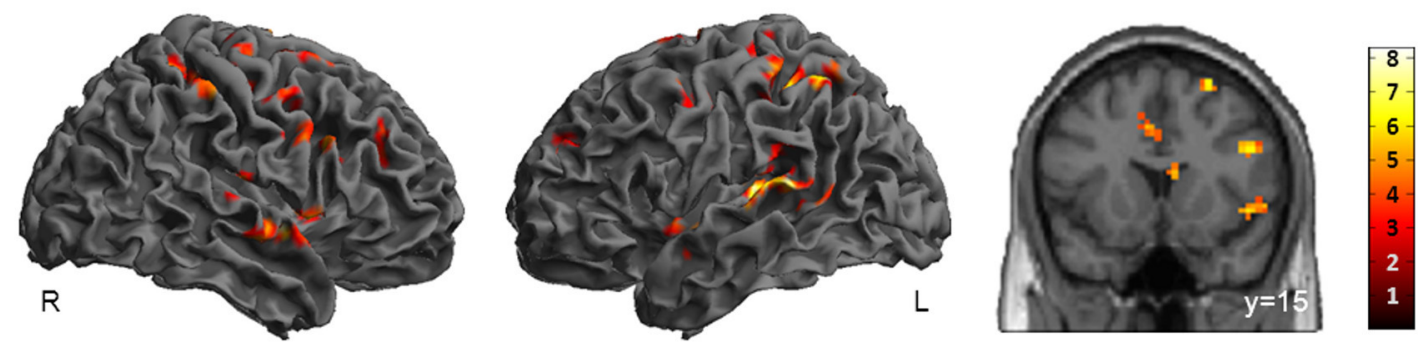

b

Positive single-trial correlations of P3 at Cz with fMRI BOLD signal

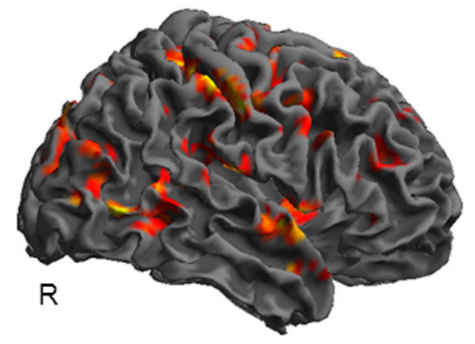

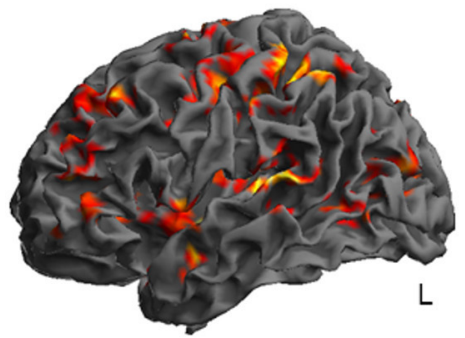
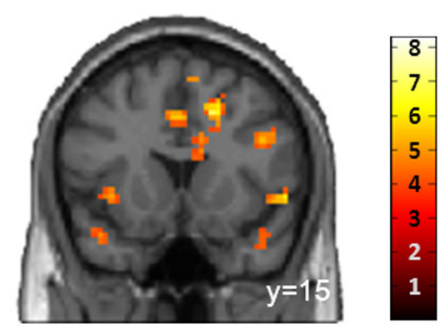

FIGURE 4 | (A) Grand average (a) and grand mean topographies at N2 latency range (b) and P3 latency range (c). Mean and 95\% confidence interval (Cl) of Nogo (black lines) and Go (green lines) are displayed in solid and dotted lines; gray bars indicate the early and late latency ranges on the group level (a). (B) Activation maps displaying the main effects of positive correlations with EEG-derived N2 (a; time window 280-340 ms after stimulus onset) and P3 (b; time window 350-570 ms after stimulus onset) regressors. These regressors were orthogonalized to onset regressors. Images are displayed in neurological order ( $L$, Left; $R$, Right), with $p<0.005$ (unc.) and $k=20$. orthogonalization, these regressors revealed those parts of the trial-to-trial fluctuation that are not captured by the onset regressors. As shown in Figure 3B, EEG-derived regressors correlated positively with fMRI BOLD signal in cortical and subcortical regions associated with response inhibition. Although no significant differences between early and late were found at the level of $p<0.05$ (FWE corrected), it can be seen that correlations with early and late EEG-derived regressors revealed overlapping but also different areas of activation in regions associated with response inhibition.
The early regressor but not the late regressors correlated positively with fMRI BOLD signal in bilateral frontal regions such as right posterior IFG (pars opercularis), right Insula/IFG (pars orbitalis), bilateral superior frontal gyrus and left precentral gyrus (adjacent to inferior frontal junction) as well as bilateral insula lobe. Subcortically positive correlations between fMRI BOLD signal and early regressor were found in right putamen, bilateral caudate nucleus and bilateral pallidum, whereas the late regressor correlated positively with left putamen. Positive correlations with both regressors but with reduced cluster size in correlations with 
A a Grand average of visual independent components (ICs)

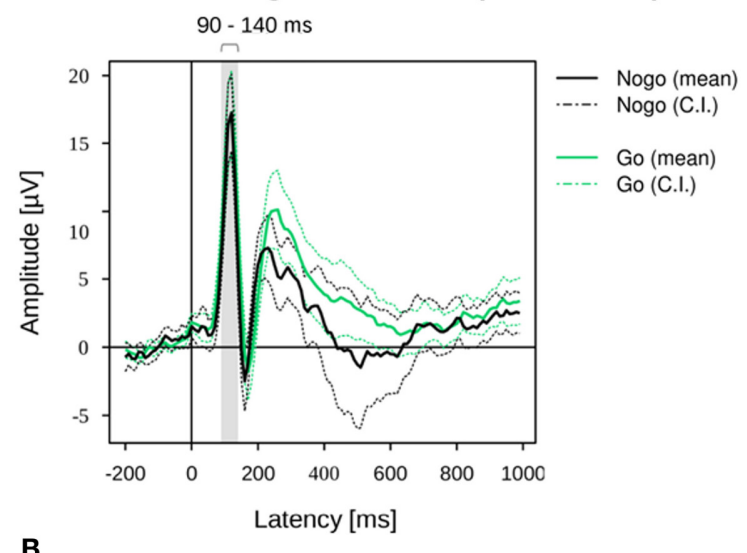

b Topography of Nogo at $120 \mathrm{~ms}$

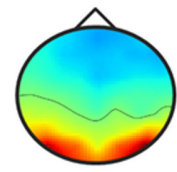

C Topography of Go at $120 \mathrm{~ms}$

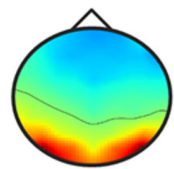

a Positive single-trial correlations of visual ICs with fMRI BOLD signal
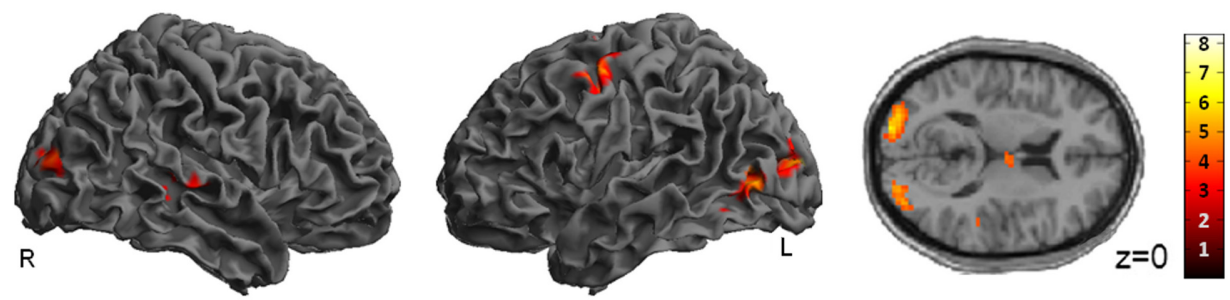

FIGURE 5 | (A) Grand averages (a) and grand mean IC topography (b) of all participants ICs which were classified as reliable related to visual processing within the latency range of 90-140 ms. Mean and 95\% confidence interval (Cl) of Nogo (black lines) and Go (green lines) are displayed in solid and dotted lines; the gray bar indicates the latency range of 90-140 ms (a). (B) Positive correlations of $\mathrm{fMRI} B O L D$ signal with EEG regressor derived from visual components (90-140 ms after stimulus onset). Images are displayed in neurological order (L, Left; $R$, Right), with $p<0.005$ (unc.) and $k=20$. late regressor were found in pre-SMA, bilateral dorso-lateral prefrontal cortex and right supramarginal gyrus/temporo-parietal junctions. Further positive correlations between fMRI BOLD signal and both EEG-derived regressors were found in left superior temporal gyrus, right precuneus, bilateral occipital regions, left hippocampus and bilateral insula lobe/amygdala.

\section{EEG/fMRI SINGLE-TRIAL ANALYSIS OF N2/P3 ERPs}

Positive and negative correlations of the AMRI BOLD signal with EEG regressors derived from $\mathrm{Cz}$ electrode at the latency ranges N2 (280-340 ms post-stimulus) and P3 (350-570 ms post-stimulus) are listed in Tables 2, 3. As these regressors were orthogonalized to onset regressors, correlations of these EEG-derived regressors with fMRI BOLD signal only revealed that part of the trial-totrial fluctuation that is not captured by the onset regressors. As shown in Figure 4B, the N2/P3 EEG-derived regressors correlated positively with $\mathrm{fMRI}$ BOLD signal in cortical and subcortical regions associated with response inhibition. Despite significant differences $(\mathrm{P} 3>\mathrm{N} 2)$ in left postcentral gyrus, left STN/thalamus and a large area stretching from cerebellar vermis/lingual gyrus to cuneus/precuneus (Table 4), it can be seen that correlations with $\mathrm{N} 2$ and P3 EEG-derived regressors revealed overlapping but also different areas of activation in regions associated with response inhibition.

The N2 regressor but not the P3 regressors correlated positively with $\mathrm{AMRI}$ BOLD signal in right posterior IFG (pars opercularis), right superior temporal gyrus and left caudate nucleus. The $\mathrm{P} 3$ regressor but not the $\mathrm{N} 2$ regressors correlated positively with fMRI BOLD signal in left anterior IFG (pars triangularis), right precentral gyrus, left middle cingulate cortex, right middle and inferior temporal regions, bilateral occipital areas and subcortical regions such as left putamen, left STN and bilateral thalamus/hippocampus. Positive correlations with both regressors but with reduced cluster size in correlations with P3 regressor were found in right Insula/IFG (pars orbitalis) and right precuneus/inferior parietal lobule. Reduced cluster size in correlations with $\mathrm{N} 2$ regressor compared to $\mathrm{P} 3$ regressor was found in left dorso-lateral prefrontal cortex, right pre-SMA and left superior temporal gyrus. The $\mathrm{N} 2$ regressor but not the $\mathrm{P} 3$ regressors correlated negatively with fMRI BOLD signal in a cluster located at the superior medial frontal gyrus and a large area stretching from central lingual gyrus and cerebellum to precuneus and calcarine gyrus, but also in smaller cortical and subcortical clusters located in pre-SMA, left middle frontal gyrus, bilateral fusiform gyri, left STN, and right Pallidum and caudate nucleus (Table 3).

Supplementary Table 1 contains a side-by-side comparison of positive fMRI BOLD correlations obtained using the new method (early/late regressors, section EEG/fMRI Single-trial Analysis of Nogo-related ICs) and the classical method (current section).

\section{EEG/fMRI SINGLE-TRIAL ANALYSIS OF VISUAL ICs}

Positive correlations of the $\mathrm{PMRI}$ BOLD signal with EEG regressors derived from IC related to visual processing (ICs with $|Z|>$ 
0.275 within the latency ranges of $90-140 \mathrm{~ms}$ post-stimulus) are listed in Table 5. Correlations of fMRI BOLD signal with EEG regressor derived from single-trial amplitudes of visual components yielded activations primarily in visual areas but also in the left premotor cortex (Figure 5B). However, significant positive correlations at the level of $p<0.05$ (FWE corrected) were found exclusively in visual areas (bilateral middle and superior occipital gyri) but not in premotor areas.

\section{DISCUSSION}

The current study aimed at a data-driven identification of correlates of trial-to-trial variability in inhibition specific neurophysiological activity in simultaneously acquired EEG and fMRI. Using data of 39 healthy participants in a visual Go/Nogo task, single trial EEG/fMRI analysis was performed based on the automated identification of inhibition-related electrophysiological ICs. This identification was done for each participant in a completely data driven way using an extended ICA (Bell and Sejnowski, 1995; Lee et al., 1999; Makeig et al., 1999, 2002). Specifically Nogo-related ICs (i.e., Nogo minus Go) were identified by $Z$-scores of stimuluslocked averages above a predefined threshold within one of two time windows.

\section{SINGLE-TRIAL EEG/fMRI ANALYSIS OF NOGO-RELATED ICs AND N2/P3} ERPs

In those participants showing reliable inhibition-related components, we were able to analyze the relationship between trialto-trial variations in these ICs and fMRI brain activity. Due to

Table 1 | Positive correlations of fMRI BOLD signal with orthogonalized EEG-derived regressors early (time window starting $200 \mathrm{~ms}$ after stimulus onset and ending with the individual median RT) and late (time window starting $100 \mathrm{~ms}$ prior to RT and ending $300 \mathrm{~ms}$ after RT).

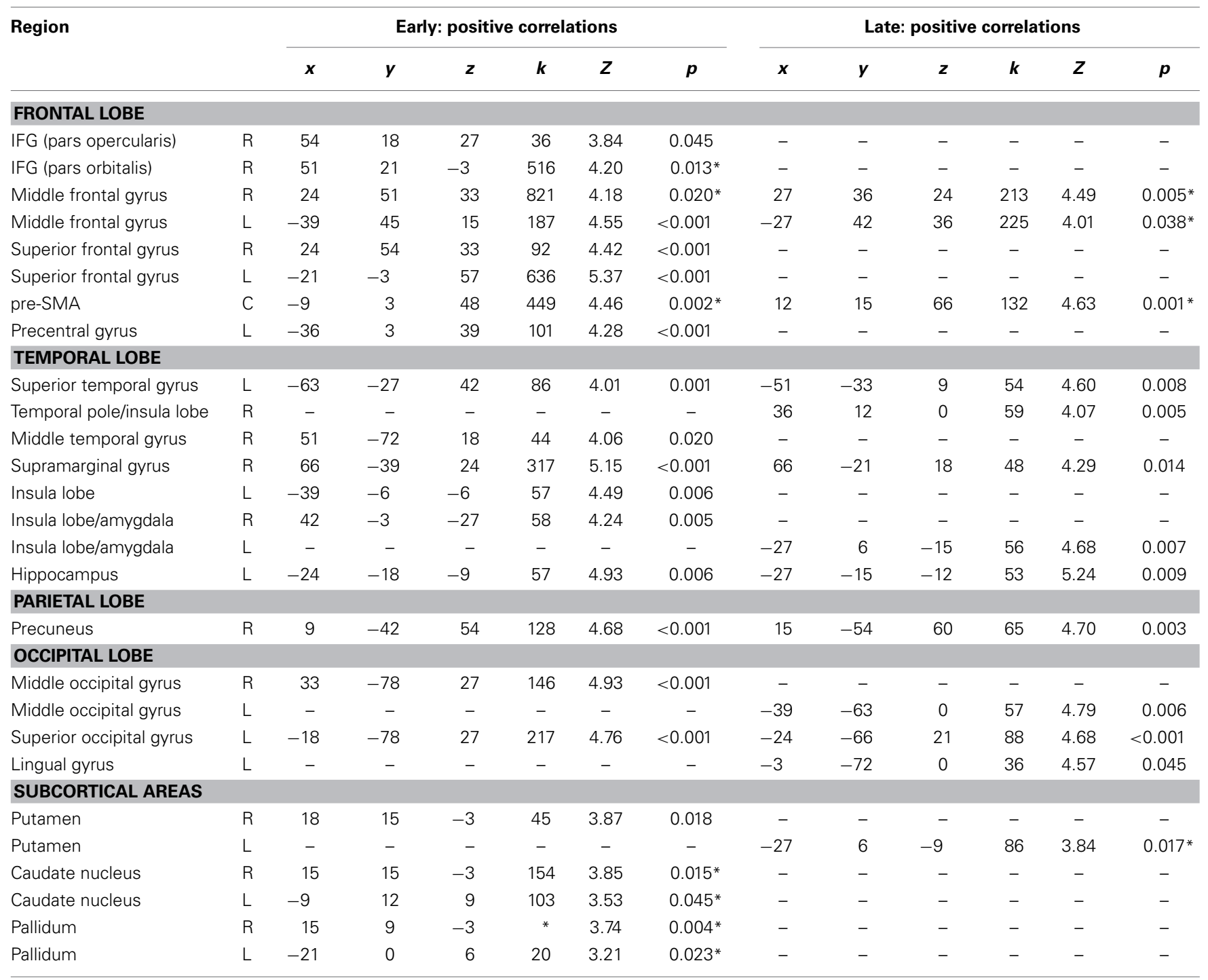

The region in which the cluster's local maximum is located in hemispheres Right (R), Left (L), or Central (C); the peak location in MNI coordinates ( $x, y$, $z)$; cluster extend in number of voxels ( $\mathrm{k}$ ); maximum Z-score; and FWE-corrected p-values (cluster level corrected, ${ }^{*}$ small volume corrected) are reported for each significantly activated cluster separately. IFG, inferior frontal cortex. SMA, supplementary motor area. 
the high temporal resolution of the EEG, we could specifically assess inhibition-related EEG activity occurring clearly before the typical RT (early time window) and inhibition-related EEG activity occurring around the typical RT (later time window; Figure 3A). The corresponding EEG-derived regressors were orthogonalized to the classical paradigm-derived onset regressors to reveal only those brain regions in which the BOLD signal is attributed genuinely to trial-to-trial fluctuations of inhibitionrelated ICs rather than to condition effects. Both, early and late EEG-derived regressors correlated positively with frontostriatal regions (right IFC, pre-SMA and basal ganglia) associated with response inhibition (Chambers et al., 2009; Aron, 2011).
Although there were no significant differences between early and late, in most areas including right Insula/IFC, right posterior IFC, premotor areas and basal ganglia, correlations of fMRI BOLD signal with the early EEG regressor were stronger than with the late EEG regressor. This indicates that the strength of positive correlations is decreasing from early to late stages of response inhibition. However, when interpreting results of single-trial EEG/fMRI analysis it should be considered that although the EEG's high temporal resolution allows extracting electrophysiological activity clearly related to different stages of neural processing, the fMRI BOLD signal's temporal resolution remains low. Accordingly, we are able to correlate electrophysiological signals generated by the

Table 2 | Positive correlations of fMRI BOLD signal with orthogonalized EEG-derived regressors N2 (280-340 ms after stimulus onset), and P3 (350-570 $\mathrm{ms}$ after stimulus onset).

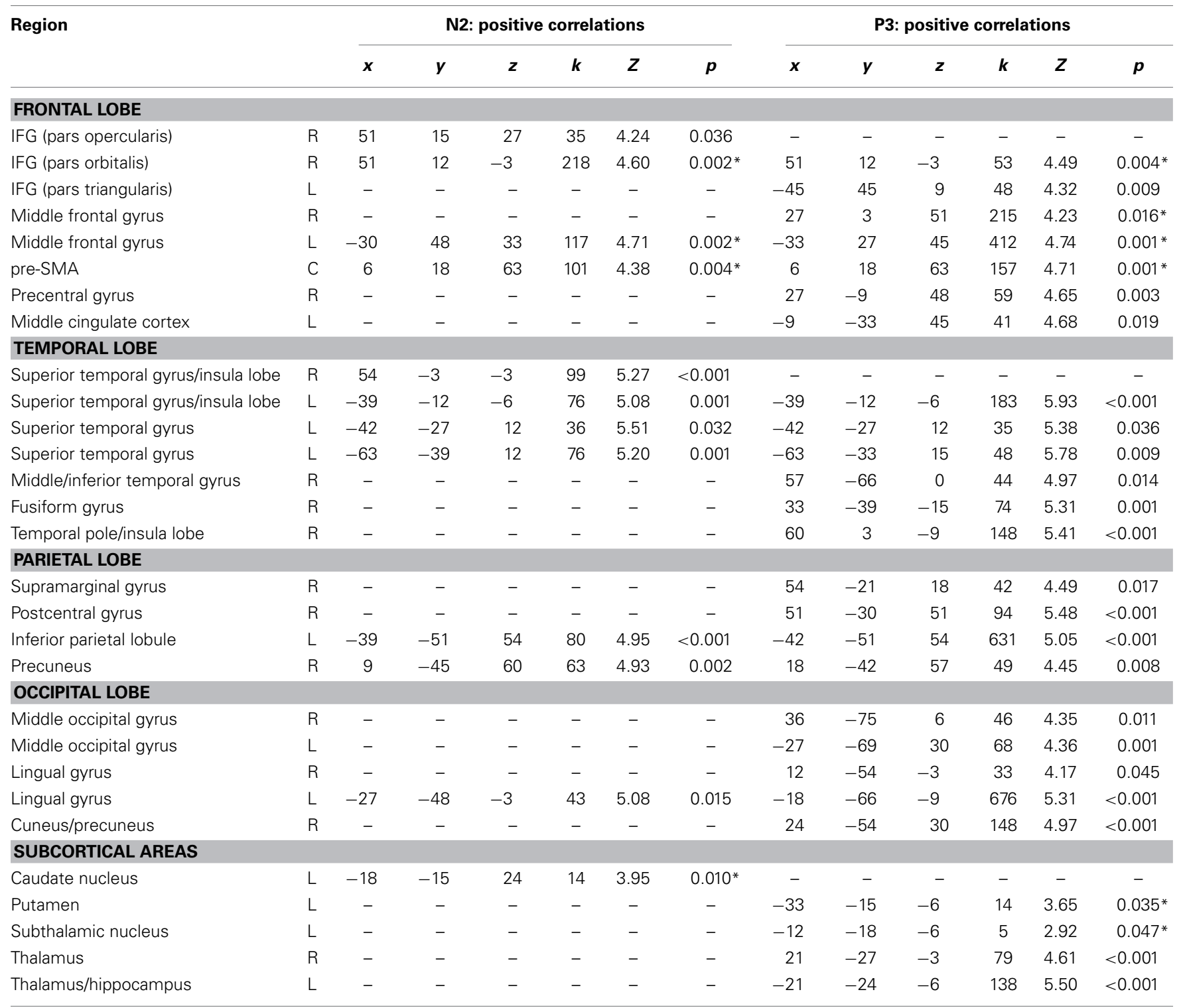

The region in which the cluster's local maximum is located in hemispheres Right (R), Left (L), or Central (C); peak location in MNI coordinates ( $x, y, z)$; cluster extend in number of voxels (k); the maximum Z-score; and FWE-corrected p-values (cluster level corrected, ${ }^{*}$ small volume corrected) are reported for each significantly activated cluster separately. IFG, inferior frontal cortex. SMA, supplementary motor area. 
brain at different stages of neural processing with the AMRI BOLD signal but we are not able to distinguish whether there is a causal relationship between a certain region and the Nogo-related activity or whether the activity of the regions are just statistically more likely to be preceded, accompanied, or followed by Nogo-related activity without a causal relationship.

Table 3 | Negative correlations of fMRI BOLD signal with orthogonalized EEG-derived regressor N2 (280-340 $\mathrm{ms}$ after stimulus onset).

\begin{tabular}{lcccccccc}
\hline Region & & \multicolumn{7}{c}{ N2: negative correlations } \\
\cline { 3 - 8 } & & $\boldsymbol{x}$ & $\boldsymbol{y}$ & $\boldsymbol{z}$ & $\boldsymbol{k}$ & $\boldsymbol{Z}$ & $\boldsymbol{p}$ \\
\hline FRONTAL LOBE & & & & & & & \\
Superior medial gyrus & $\mathrm{L}$ & -6 & 60 & 3 & 121 & 5.00 & $<0.001$ \\
Middle frontal gyrus & $\mathrm{L}$ & -27 & 54 & 9 & 48 & 4.26 & $0.013^{*}$ \\
pre-SMA & $\mathrm{C}$ & 0 & 18 & 54 & 73 & 3.99 & $0.020^{*}$ \\
TEMPORAL LOBE & & & & & & & \\
Fusiform gyrus & $\mathrm{R}$ & 27 & -42 & -12 & 88 & 5.21 & $<0.001$ \\
Fusiform gyrus & $\mathrm{L}$ & -24 & -45 & -15 & 47 & 5.17 & 0.010 \\
Angular gyrus & $\mathrm{L}$ & -42 & -75 & 39 & 48 & 4.22 & 0.009 \\
OCCIPITAL LOBE & & & & & & & \\
Lingual gyrus/cerebellum & $\mathrm{R}$ & 9 & -54 & -15 & 156 & 4.52 & $<0.001$ \\
SUBCORTICAL AREAS & & & & & & & \\
Caudate nucleus & $\mathrm{R}$ & 12 & -3 & 18 & 31 & 3.60 & $0.041^{*}$ \\
Pallidum & $\mathrm{R}$ & 15 & 3 & 3 & 10 & 3.68 & $0.005^{*}$ \\
Subthalamic nucleus & $\mathrm{L}$ & -12 & -18 & -9 & 8 & 3.16 & $0.021^{*}$ \\
\hline
\end{tabular}

The region in which the cluster's local maximum is located in hemispheres right $(R)$, left $(L)$, or central $(C)$; peak location in $M N I$ coordinates $(x, y, z)$; cluster extend in number of voxels ( $k$ ); the maximum Z-score; and FWE-corrected $p$ values (cluster level corrected, " small volume corrected) are reported for each significantly activated cluster separately. SMA, supplementary motor area.

Table 4 | Brain regions significantly stronger correlated with P3 single-trial amplitude values (350-570 $\mathrm{ms}$ after stimulus onset) than with N2 single-trial amplitude values $(280-340 \mathrm{~ms}$ after stimulus onset).

\begin{tabular}{|c|c|c|c|c|c|c|c|}
\hline \multirow{2}{*}{\multicolumn{2}{|c|}{ Region }} & \multicolumn{6}{|c|}{ P3 > N2 } \\
\hline & & $\boldsymbol{x}$ & $y$ & $z$ & $\boldsymbol{k}$ & $Z$ & $p$ \\
\hline \multicolumn{8}{|l|}{ PARIETAL LOBE } \\
\hline Postcentral gyrus & $\mathrm{L}$ & -39 & -24 & 54 & 91 & 3.45 & 0.029 \\
\hline \multicolumn{8}{|l|}{ OCCIPITAL LOBE } \\
\hline Lingual/calcarine gyrus & C & 0 & -63 & 12 & 414 & 4.53 & $<0.001$ \\
\hline \multicolumn{8}{|c|}{ SUBCORTICAL AREAS } \\
\hline Subthalamic nucleus & $\mathrm{L}$ & -12 & -18 & -6 & 11 & 3.49 & 0.006 \\
\hline Thalamus & $\mathrm{L}$ & -18 & -24 & -6 & 88 & 3.61 & 0.034 \\
\hline
\end{tabular}

The region in which the cluster's local maximum is located in hemispheres Left $(L)$ or Central (C); peak location in MNI coordinates ( $x, y, z)$; cluster extend in number of voxels ( $k$ ); maximum Z-score; and FWE-corrected p-values (cluster level corrected, *small volume corrected) are reported for each significantly activated cluster separately.
Single-trial correlation of N2/P3 amplitude values with fMRI BOLD signal was computed for the same 22 participants as the single-trial EEG/fMRI analysis of Nogo-related ICs. The N2dervied EEG regressor correlated negatively with regions associated with the default mode network (precuneus and superior medial cortex) (Raichle et al., 2001; Buckner et al., 2008) but also with areas in pre-SMA, middle frontal gyrus and basal ganglia. To some degree, these results are consistent with Baumeister et al. (2014), who found negative correlations of increased N2 amplitudes in right middle frontal gyrus, bilateral middle temporal and fusiform gyri but also in regions associated with the default mode network (right precuneus, bilateral superior temporal gyrus and right medial frontal gyrus). As discussed by Baumeister et al. (2014), this might indicate an association between increasing N2 amplitudes and deactivation of the default mode network.

Both, N2- and P3-derived EEG regressors correlated positively with $\mathrm{fMRI} B \mathrm{BLD}$ signal in fronto-striatal regions associated with response inhibition (right IFC, pre-SMA and basal ganglia), but also in distributed areas located in temporal and parietal lobule. Except for right IFC the degree of positive correlations with N2/P3-derived EEG regressors increased from N2 to P3, whereas in IC-based EEG/fMRI analysis fewer regions were positively correlated with the late EEG regressor relative to the early EEG regressor. The latter may indicate that regions relevant for a successful response inhibition are up-regulated at an early stage of response inhibition but not at the later stage of response inhibition. This seems to be reasonable as it could be expected that regions essential for withholding a prepotent motor response are activated prior to the time point when the Go response would be executed. In contrast to this, N2/P3 single-trial amplitude values seem to correlate with a mixture of network parts associated with response inhibition, attentional processing or response monitoring.

When comparing both approaches (i.e., early/late vs. N2/P3) it can be seen that the activation pattern yielded by the early EEG regressor and the N2 EEG regressor are largely deviating. This

Table 5 | Positive correlations of fMRI BOLD signal EEG-regressor derived from visual response (time window starting $90 \mathrm{~ms}$ after stimulus onset and ending $140 \mathrm{~ms}$ after stimulus onset).

\begin{tabular}{llrrrrrrr}
\hline Region & & \multicolumn{7}{c}{ Visual components } \\
\cline { 3 - 8 } & & $\boldsymbol{x}$ & $\boldsymbol{y}$ & $\boldsymbol{z}$ & $\boldsymbol{k}$ & $\boldsymbol{Z}$ & $\boldsymbol{p}$ \\
\hline OCCIPITAL LOBE & & & & & & & \\
Superior occipital gyrus & $\mathrm{R}$ & 24 & -93 & 12 & 42 & 3.65 & $0.037^{*}$ \\
Superior occipital gyrus & $\mathrm{L}$ & -15 & -93 & 12 & 70 & 4.22 & $0.003^{*}$ \\
Middle occipital gyrus & $\mathrm{R}$ & 33 & -87 & 15 & 114 & 3.88 & $0.021^{*}$ \\
Middle occipital gyrus & $\mathrm{L}$ & -21 & -93 & 9 & 55 & 5.25 & 0.019 \\
Middle occipital gyrus & $\mathrm{L}$ & -42 & -69 & 3 & 59 & 4.69 & 0.014
\end{tabular}

The region in which the cluster's local maximum is located in hemispheres Right $(R)$ or Left $(L)$; peak location in MNI coordinates $(x, y, z)$; cluster extend in number of voxels (k); maximum Z-score; and FWE-corrected p-values (cluster level corrected, * small volume corrected) are reported for each significantly activated cluster separately. 
might be related to fundamental differences in both approaches. While the early and late EEG regressors were constructed exclusively of participant-specific components which are reliably differentiating between different task conditions at the respective latency range, the N2/P3 ERPs were defined on the group level at the latency ranges and EEG site with the most pronounced Nogo effect. Repeated measure ANOVA with the factors condition (Go and Nogo) and ERP (N2 and P3) revealed a significant condition $\mathrm{x}$ ERP interaction $\left[F_{(1,21)}=61.516, p<0.001\right]$ on mean amplitude values. However, post hoc test revealed that mean amplitudes of P3 but not of N2 were significantly different between Go and Nogo which is in line with Baumeister et al. (2014), who also reported significant differences for P3 amplitude values but not for N2 amplitude values. The prominent difference between correlations of the $\mathrm{AMRI}$ BOLD signal with the early EEG regressor or the N2 EEG regressor might be related to the fact that the early ICs are reliably task-discriminating at the respective latency range and thus more sensitive to the Nogo condition, while the N2 seems to be less specific to the task condition.

In conclusion, the deviating results between ERP-based N2/P3 and IC-based early/late single-trial correlations are probably related to the fundamentally different approaches of selecting the EEG features used for single-trial correlations. Following Baumeister et al. (2014), for each participant the mean single-trial amplitude values of $\mathrm{N} 2$ and $\mathrm{P} 3$ were extracted from $\mathrm{Cz}$ electrode at the latency ranges $280-340$ and $350-570 \mathrm{~ms}$ after stimulus onset. These time windows were chosen as they reflected best the Nogo-N2 and Nogo-P3 effects for the entire group (Figure 4A). However, these fixed time windows were determined on the group level which is insensitive to inter-individual variability as they were observable for example in the participant's median RT (ranging from 322.81 to $487.94 \mathrm{~ms}$ ). As it is known that such phenotypes exist even for simple reaction time paradigms, inter-individual differences constitute valuable information when analyzing more complex cognitive functions (Kanai and Rees, 2011). Thus, inter-individual differences make it necessary to verify intra-individually the presence of certain components prior to including them into group-level analyses. Therefore, we developed an analysis procedure that does not build exclusively on N2/P3 effects, but classifies and selects task- and participantspecific electrophysiological components in a completely data driven manner. For every single participant, the algorithm identifies those participant-specific components which are differentiating best between the different task conditions at the specific latency range (Figure 3A). Thus, in contrast to N2/P3 ERPs which were defined on the group level (Figure 4A), early and late regressors were constructed of functionally characterized ICs.

\section{EEG/fMRI SINGLE-TRIAL ANALYSIS OF VISUAL ICs}

We introduced an algorithm that allows for selecting Nogorelated ICs in an automated procedure; however, the fact that the algorithm could identify Nogo-related ICs only in about half of the participants may question the validity of the algorithm. Thus, to test whether our IC selection method is generalizable and usable beyond Nogo-related IC detection, we modified the algorithm so that ICs associated with the visual responses can be detected. Single-trial amplitudes of ICs related to visual processing (i.e., $|Z|>0.275$ within the latency ranges of 90-140 ms post-stimulus) correlated positively with fMRI BOLD signal in left inferior occipital gyrus as well as bilateral middle and superior occipital gyri.

The results are consistent with Fuglø et al. (2012) who found positive correlations of visual components with fMRI BOLD responses in primary visual cortex and middle occipital gyrus. However, Fuglø et al. (2012) employed a block design with checkerboard stimulus blocks alternating with blocks without stimuli, while continuously estimating VEP amplitudes. Therefore, the resulting regressor necessarily follows the stimulation design to a larger degree. EEG-fMRI correlations from experiments in which either a constant stimulus is repeated or stimuli matched for physical properties such as size, complexity and luminance are presented must be viewed from a different perspective. Variability observed across such stimuli may either reflect early discriminative activity for different stimuli or spontaneous fluctuations in brain state. A study following a spontaneous fluctuation design (visual oddball with motor responses) comparable to the current one revealed positive correlations of visual components with fMRI BOLD signal not only in regions of the visual dorsal stream but also in medial frontal and precentral gyri (Warbrick et al., 2013). In addition to visual cortex activity our approach also revealed medial frontal precentral activity patterns comparable to Warbrick et al. (2013). Thus, despite of different task settings and the fact that Warbrick et al. (2013) used a selection procedure based on a-priori defined ERP components (P1 and N1), whereas we selected ICs related to visual processing in a purely data-driven approach, the resultant correlations between EEG-derived regressors and $\mathrm{AMRI}$ BOLD responses are quite similar. This illustrates that beyond of detecting Nogorelated ICs our algorithm is also able to detect ICs related to visual responses in the same data set.

\section{LIMITATIONS}

As a result of IC selection only about half of participants could be included into the single-trial EEG/fMRI data analysis. As such these results of temporal signal evolution in response inhibition may not be generalizable. Moreover, the fact that Nogo-related ICs were not identified in one half of the participants suggests that correlations using these restrictive IC selection criteria can only be determined for part of the initial population of a study. On the other hand, the same algorithm was able to detect ICs related to visual responses in 37 of 39 participants, which argues against a principal failure of the algorithm. Thus, the finding that certain Nogo-related components are not present in every participant may give an indication of substantial inter-individual differences as state or trait related differences in cognitive strategy when performing the task, illustrating the sensitivity and specificity of the algorithm for detection of different event related responses.

\section{CONCLUSION}

Using EEG-derived regressors based on single-trial amplitude variability of Nogo-related ICs selected with respect to different time windows allows visualizing the evolution of brain processes during motor inhibition. Furthermore, by classifying and selecting ICs intra-individually our approach takes account of known 
inter-individual differences in neural processing. In line with existing automated approaches (Goldman et al., 2009; Wessel and Ullsperger, 2011) we used an algorithm that allows for selecting task-related ICs in an automated procedure. As is the case in the COMPASS-approach (Wessel and Ullsperger, 2011), our approach uses an automated IC selection procedure but is independent of ERP templates, which was one of the major drawbacks of COMPASS. This can be achieved by using an algorithm that automatically selects inhibition-specific ICs with significantly increased amplitude during Nogo trials relative to Go trials. Additionally, these ICs were automatically classified depending on whether the latency range of reliably Nogo-related activity occurred early or late relative to median correct Go RT. This is partially comparable to the approach of Goldman et al. (2009) but without the drawback of just being able to extract one component per time window. As our method is independent of a priori defined ERPs, we suggest that this approach of using functionally defined components could be used for EEG features other than event-related transient responses. Although not tested yet, one possible application would be to use it in the context of background rhythms. In this case, one could selectively use those components that are characterized by increased spectral power in one condition relative to another condition.

\section{ACKNOWLEDGMENTS}

This work was supported by the Federal Ministry of Education and Research Grant 01GW0730 (Alexandra Sebastian, Klaus Lieb, and Oliver Tüscher) and by the Focus Program Translational Neuroscience Johannes-Gutenberg-University Mainz (Lena Schmüser, PhD-Fellowship). The authors would like to thank the Freiburg Brain Imaging Center and Volkmar Glauche for continuous support, and Carlos Baldermann, Birthe Gerdes, Julian Geisshardt, Marina Pohl, and Tanja Schmitt for help with data acquisition and analyses.

\section{SUPPLEMENTARY MATERIAL}

The Supplementary Material for this article can be found online at: http://www.frontiersin.org/journal/10.3389/fnins.2014. 00175/abstract

\section{REFERENCES}

Allen, P. J., Josephs, O., and Turner, R. (2000). A method for removing imaging artifact from continuous eeg recorded during functional MRI. Neuroimage 12, 230-239. doi: 10.1006/nimg.2000.0599

Arieli, A., Sterkin, A., Grinvald, A., and Aertsen, A. (1996). Dynamics of ongoing activity: explanation of the large variability in evoked cortical responses. Science 273, 1868-1871. doi: 10.1126/science.273.5283.1868

Aron, A. R. (2011). From reactive to proactive and selective control: developing a richer model for stopping inappropriate responses. Biol. Psychiatry 69, e55-e68. doi: 10.1016/j.biopsych.2010.07.024

Aron, A. R., and Poldrack, R. A. (2006). Cortical and subcortical contributions to stop signal response inhibition: role of the subthalamic nucleus. J. Neurosci. 26, 2424-2433. doi: 10.1523/JNEUROSCI.4682-05.2006

Bagshaw, A. P., and Warbrick, T. (2007). Single trial variability of EEG and fMRI responses to visual stimuli. NeuroImage 38, 280-292. doi: 10.1016/j.neuroimage.2007.07.042

Baumeister, S., Hohmann, S., Wolf, I., Plichta, M. M., Rechtsteiner, S., Zangl, M., et al. (2014). Sequential inhibitory control processes assessed through simultaneous EEG-fMRI. Neuroimage 94, 349-359. doi: 10.1016/j.neuroimage.2014. 01.023
Bell, A. J., and Sejnowski, T. J. (1995). An information-maximization approach to blind separation and blind deconvolution. Neural Comput. 7, 1129-1159. doi: 10.1162/neco.1995.7.6.1129

Bénar, C.-G., Schön, D., Grimault, S., Nazarian, B., Burle, B., Roth, M., et al. (2007). Single-trial analysis of oddball event-related potentials in simultaneous EEGfMRI. Hum. Brain Mapp. 28, 602-613. doi: 10.1002/hbm.20289

Bland, A., Mushtaq, F., and Smith, D. V. (2011). Exploiting trial-to-trial variability in multimodal experiments. Front. Hum. Neurosci. 5:80. doi: $10.3389 /$ fnhum. 2011.00080

Bregadze, N., and Lavric, A. (2006). ERP differences with vs. without concurrent fMRI. Int. J. Psychophysiol. 62, 54-59. doi: 10.1016/j.ijpsycho.2006. 01.010

Buckner, R. L., Andrews-Hanna, J. R., and Schacter, D. L. (2008). The brain's default network. Ann. N.Y. Acad. Sci. 1124, 1-38. doi: 10.1196/annals. 1440.011

Chambers, C. D., Garavan, H., and Bellgrove, M. A. (2009). Insights into the neural basis of response inhibition from cognitive and clinical neuroscience. Neurosci. Biobehav. Rev. 33, 631-646. doi: 10.1016/j.neubiorev.2008.08.016

Debener, S., Ullsperger, M., Siegel, M., and Engel, A. K. (2006). Single-trial EEGfMRI reveals the dynamics of cognitive function. Trends Cogn. Sci. 10, 558-563. doi: 10.1016/j.tics.2006.09.010

Debener, S., Ullsperger, M., Siegel, M., Fiehler, K., von Cramon, D. Y., and Engel, A. K. (2005). Trial-by-trial coupling of concurrent electroencephalogram and functional magnetic resonance imaging identifies the dynamics of performance monitoring. J. Neurosci. 25, 11730-11737. doi: 10.1523/JNEUROSCI.328605.2005

Di Russo, F., Martínez, A., Sereno, M. I., Pitzalis, S., and Hillyard, S. A. (2002). Cortical sources of the early components of the visual evoked potential. Hum. Brain Mapp. 15, 95-111. doi: 10.1002/hbm.10010

Di Russo, F., Pitzalis, S., Spitoni, G., Aprile, T., Patria, F., Spinelli, D., et al. (2005). Identification of the neural sources of the pattern-reversal VEP. Neuroimage 24, 874-886. doi: 10.1016/j.neuroimage.2004.09.029

Eichele, T., Calhoun, V. D., and Debener, S. (2009). Mining EEG-fMRI using independent component analysis. Int. J. Psychophysiol. 73, 53-61. doi: 10.1016/j.ijpsycho.2008.12.018

Eichele, T., Specht, K., Moosmann, M., Jongsma, M. L. A., Quiroga, R. Q., Nordby, H., et al. (2005). Assessing the spatiotemporal evolution of neuronal activation with single-trial event-related potentials and functional MRI. Proc. Natl. Acad. Sci. U.S.A. 102, 17798-17803. doi: 10.1073/pnas. 0505508102

Feige, B. (1999). Oscillatory Brain Activity and its Analysis on the Basis of MEG and EEG. Münster: Waxmann. ISBN: 9783893256983

Feige, B., Scheffler, K., Esposito, F., Di Salle, F., Hennig, J., and Seifritz, E. (2005). Cortical and subcortical correlates of electroencephalographic alpha rhythm modulation. J. Neurophysiol. 93, 2864-2872. doi: 10.1152/jn.007 21.2004

Fontanini, A., and Katz, D. B. (2008). Behavioral states, network states, and sensory response variability. J. Neurophysiol. 100, 1160-1168. doi: $10.1152 /$ jn. 90592.2008

Fuglø, D., Pedersen, H., Rostrup, E., Hansen, A. E., and Larsson, H. B. W. (2012). Correlation between single-trial visual evoked potentials and the blood oxygenation level dependent response in simultaneously recorded electroencephalography-functional magnetic resonance imaging. Magn. Reson. Med. 68, 252-260. doi: 10.1002/mrm.23227

Goldman, R. I., Wei, C.-Y., Philiastides, M. G., Gerson, A. D., Friedman, D., Brown, T. R., et al. (2009). Single-trial discrimination for integrating simultaneous EEG and fMRI: Identifying cortical areas contributing to trial-totrial variability in the auditory oddball task. Neuroimage 47, 136-147. doi: 10.1016/j.neuroimage.2009.03.062

Huster, R. J., Debener, S., Eichele, T., and Herrmann, C. S. (2012). Methods for simultaneous EEG-fMRI: an introductory review. J. Neurosci. 32, 6053-6060. doi: 10.1523/JNEUROSCI.0447-12.2012

Johnson, J. S., and Olshausen, B. A. (2003). Timecourse of neural signatures of object recognition. J. Vis. 3, 499-512. doi: 10.1167/3.7.4

Juckel, G., Karch, S., Kawohl, W., Kirsch, V., Jäger, L., Leicht, G., et al. (2012). Age effects on the P300 potential and the corresponding fMRI BOLD-signal. Neuroimage 60, 2027-2034. doi: 10.1016/j.neuroimage.2012.02.019

Kanai, R., and Rees, G. (2011). The structural basis of inter-individual differences in human behaviour and cognition. Nat. Rev. Neurosci. 12, 231-242. doi: $10.1038 / \mathrm{nrn} 3000$ 
Karch, S., Jäger, L., Karamatskos, E., Graz, C., Stammel, A., Flatz, W., et al. (2008). Influence of trait anxiety on inhibitory control in alcohol-dependent patients: simultaneous acquisition of ERPs and BOLD responses. J. Psychiatr. Res. 42, 734-745. doi: 10.1016/j.jpsychires.2007.07.016

Karch, S., Thalmeier, T., Lutz, J., Cerovecki, A., Opgen-Rhein, M., Hock, B., et al. (2010). Neural correlates (ERP/fMRI) of voluntary selection in adult ADHD patients. Eur. Arch. Psychiatry Clin. Neurosci. 260, 427-440. doi: 10.1007/s00406-009-0089-y

Kelly, A. M. C., Uddin, L. Q., Biswal, B. B., Castellanos, F. X., and Milham, M. P. (2008). Competition between functional brain networks mediates behavioral variability. Neuroimage 39, 527-537. doi: 10.1016/j.neuroimage.2007.08.008

Lavric, A., Bregadze, N., and Benattayallah, A. (2011). Detection of experimental ERP effects in combined EEG-fMRI: evaluating the benefits of interleaved acquisition and Independent component analysis. Clin. Neurophysiol. 122, 267-277. doi: 10.1016/j.clinph.2010.06.033

Ledberg, A., Montagnini, A., Coppola, R., and Bressler, S. L. (2012). Reduced variability of ongoing and evoked cortical activity leads to improved behavioral performance. PLOS ONE 7:e43166. doi: 10.1371/journal.pone.0043166

Lee, T.-W., Girolami, M., and Sejnowski, T. J. (1999). Independent component analysis using an extended infomax algorithm for mixed subgaussian and supergaussian sources. Neural Comput. 11, 417-441. doi: $10.1162 / 089976699300016719$

Lutz, A., Lachaux, J.-P., Martinerie, J., and Varela, F. J. (2002). Guiding the study of brain dynamics by using first-person data: synchrony patterns correlate with ongoing conscious states during a simple visual task. Proc. Natl. Acad. Sci. U.S.A. 99, 1586-1591. doi: 10.1073/pnas.032658199

MacDonald, S. W. S., Nyberg, L., and Bäckman, L. (2006). Intra-individual variability in behavior: links to brain structure, neurotransmission and neuronal activity. Trends Neurosci. 29, 474-480. doi: 10.1016/j.tins.2006.06.011

Makeig, S., Westerfield, M., Jung, T.-P., Covington, J., Townsend, J., Sejnowski, T. J., et al. (1999). Functionally independent components of the late positive event-related potential during visual spatial attention. J. Neurosci. 19, 2665-2680.

Makeig, S., Westerfield, M., Jung, T.-P., Enghoff, S., Townsend, J., Courchesne, E., et al. (2002). Dynamic brain sources of visual evoked responses. Science 295, 690-694. doi: 10.1126/science. 1066168

Mobascher, A., Brinkmeyer, J., Warbrick, T., Musso, F., Wittsack, H. J., Saleh, A., et al. (2009). Laser-evoked potential P2 single-trial amplitudes covary with the fMRI BOLD response in the medial pain system and interconnected subcortical structures. Neuroimage 45, 917-926. doi: 10.1016/j.neuroimage.2008.12.051

Mulert, C., Seifert, C., Leicht, G., Kirsch, V., Ertl, M., Karch, S., et al. (2008). Single-trial coupling of EEG and fMRI reveals the involvement of early anterior cingulate cortex activation in effortful decision making. Neuroimage 42 , 158-168. doi: 10.1016/j.neuroimage.2008.04.236

Novitskiy, N., Ramautar, J. R., Vanderperren, K., De Vos, M., Mennes, M., Mijovic, B., et al. (2011). The BOLD correlates of the visual P1 and N1 in single-trial analysis of simultaneous EEG-fMRI recordings during a spatial detection task. Neuroimage 54, 824-835. doi: 10.1016/j.neuroimage.2010.09.041

Oldfield, R. C. (1971). The assessment and analysis of handedness: the Edinburgh inventory. Neuropsychologia 9, 97-113. doi: 10.1016/0028-3932(71)90067-4

Raichle, M. E., MacLeod, A. M., Snyder, A. Z., Powers, W. J., Gusnard, D. A., and Shulman, G. L. (2001). A default mode of brain function. Proc. Natl. Acad. Sci. U.S.A. 98, 676-682. doi: 10.1073/pnas.98.2.676
Scheibe, C., Ullsperger, M., Sommer, W., and Heekeren, H. R. (2010). Effects of parametrical and trial-to-trial variation in prior probability processing revealed by simultaneous electroencephalogram/functional magnetic resonance imaging. J. Neurosci. 30, 16709-16717. doi: 10.1523/JNEUROSCI.3949-09.2010

Sebastian, A., Baldermann, C., Feige, B., Katzev, M., Scheller, E., Hellwig, B., et al. (2013a). Differential effects of age on subcomponents of response inhibition. Neurobiol. Aging 34, 2183-2193. doi: 10.1016/j.neurobiolaging.2013.03.013

Sebastian, A., Gerdes, B., Feige, B., Klöppel, S., Lange, T., Philipsen, A., et al. (2012). Neural correlates of interference inhibition, action withholding and action cancelation in adult ADHD. Psychiatry Res. 202, 132-141. doi: 10.1016/j.pscychresns.2012.02.010

Sebastian, A., Pohl, M. F., Klöppel, S., Feige, B., Lange, T., Stahl, C., et al. (2013b). Disentangling common and specific neural subprocesses of response inhibition. Neuroimage 64, 601-615. doi: 10.1016/j.neuroimage.2012.09.020

Thesen, S., Heid, O., Mueller, E., and Schad, L. R. (2000). Prospective acquisition correction for head motion with image-based tracking for real-time fMRI. Magn. Reson. Med. 44, 457-465. doi: 10.1002/1522-2594(200009)44:3<457:: AID-MRM17>3.0.CO;2-R

Warbrick, T., Arrubla, J., Boers, F., Neuner, I., and Shah, N. J. (2013). Attention to detail: why considering task demands is essential for single-trial analysis of BOLD correlates of the visual P1 and N1. J. Cogn. Neurosci. 26, 529-542. doi: 10.1162/jocn_a_00490

Warbrick, T., Mobascher, A., Brinkmeyer, J., Musso, F., Richter, N., Stoecker, T., et al. (2009). Single-trial P3 amplitude and latency informed event-related fMRI models yield different BOLD response patterns to a target detection task. Neuroimage 47, 1532-1544. doi: 10.1016/j.neuroimage.2009.05.082

Wessel, J. R., and Ullsperger, M. (2011). Selection of independent components representing event-related brain potentials: a data-driven approach for greater objectivity. Neuroimage 54, 2105-2115. doi: 10.1016/j.neuroimage.2010.10.033

Wittchen, H.-U., Zaudig, M., and Frydrich, T. (1997). Strukturiertes Klinisches Interview für DSM-IV (SKID-I und SKID-II). Göttingen: Hogrefe.

Zaitsev, M., Hennig, J., and Speck, O. (2004). Point spread function mapping with parallel imaging techniques and high acceleration factors: fast, robust, and flexible method for echo-planar imaging distortion correction. Magn. Reson. Med. 52, 1156-1166. doi: 10.1002/mrm.20261

Conflict of Interest Statement: The authors declare that the research was conducted in the absence of any commercial or financial relationships that could be construed as a potential conflict of interest.

Received: 27 March 2014; accepted: 05 June 2014; published online: 01 July 2014. Citation: Schmüser L, Sebastian A, Mobascher A, Lieb K, Tüscher O and Feige B (2014) Data-driven analysis of simultaneous EEG/fMRI using an ICA approach. Front. Neurosci. 8:175. doi: 10.3389/fnins.2014.00175

This article was submitted to Brain Imaging Methods, a section of the journal Frontiers in Neuroscience.

Copyright (C) 2014 Schmüser, Sebastian, Mobascher, Lieb, Tüscher and Feige. This is an open-access article distributed under the terms of the Creative Commons Attribution License (CC BY). The use, distribution or reproduction in other forums is permitted, provided the original author(s) or licensor are credited and that the original publication in this journal is cited, in accordance with accepted academic practice. No use, distribution or reproduction is permitted which does not comply with these terms. 